\title{
Droperidol in cataract surgery
}

\author{
J. A. MENON
}

Department of Ophthalmology, Barnet General Hospital, Herts

The fact that so many techniques are available to surgeons and anaesthetists for cataract extraction, which is one of the most commonly-performed intraocular procedures, bears out the opinion of Stallard (1965) that "the ideal local and general anaesthetic for eye surgery has yet to be found".

An important factor in cataract surgery is relaxation of the extraocular muscles, especially the orbicularis oculi, and for this reason general anaesthesia is used almost exclusively in many centres. But general anaesthesia has complications of its own and, apart from such minor difficulties as coughing and expectoration after tracheal intubation, ocular akinesia is not maintained in the postoperative period when the patient may be restless.

A possible complication of local anaesthesia is retrobulbar haemorrhage but, with careful handling, this is a rare hazard, and the main problem is to keep the patient relaxed and cooperative, without making sudden violent movements.

Laborit and Huguenard (I954) introduced the concept of "hibernation anaesthesia" and suggested the use of a mixture of pethidine, promethazine, and chlorpromazine, the so-called "lytic cocktail". Intravenous injection of potent phenothiazines, however, may produce marked and long-lasting cardiovascular instability in elderly patients and the anti-emetic effect of these drugs may be counteracted by the nausea consequent on sudden hypotension.

Among the various analogues of pethidine which have recently been synthesized, phenoperidine hydrochloride has been found to be both more potent and more emetic, and to produce greater respiratory depression than its precursors (Rollason and Sutherland, 1963). To counteract the emetic effects of this useful drug it is often given in combination with one of the butyrophenones (Brown, I964), of which the best known is dehydrobenzperidol (droperidol). These drugs are potent tranquillizers and the resulting combination produces a condition known as "neuroleptanalgesia". Unfortunately the belief has arisen that, in order to produce this state of neuroleptanalgesia, these two classes of drug must be used together in the combinations suggested by the manufacturers; this misconception has caused this technique of producing a state of tranquillity with amnesia and analgesia ideal for ocular surgery in the aged, to be deprecated by some surgeons and anaesthetists as unreliable and dangerous. Any degree of intravenous overdosage with a powerful respiratory depressant such as phenoperidine (a drug used specifically for this effect in ventilator therapy) (Moran and Marshall, r966) can rapidly produce airway obstruction, desaturation, and venous engorgement; but systemic analgesia is an important adjunct in cataract surgery under local anaesthesia, because, for example, many elderly arthritic patients cannot lie still for any length of time on a hard operating table, despite the efficacy of the local anaesthesia or the gentleness of the surgeon. 
When intravenous, morphine-like analgesics are not used and reliance is placed solely on a powerful tranquillizing agent such as droperidol, alarming psychic phenomena have arisen with persistent postoperative disorientation (Nilsson and Janssen, I96I). These side-effects are not encountered, however, below a certain dose level or in the presence of adequate sedation with phenothiazines. Furthermore, droperidol is known to have $\alpha$-blocking (anti-noradrenalin) properties which could cause undesirable haemodynamic effects in susceptible patients (Holderness, Chase, and Dripps, I963). Despite these theoretical and reported objections, it was felt that a clinical trial of neuroleptanalgesia with local anaesthesia and akinesia in an unselected series of cases admitted to a general hospital for lens extraction would be valuable. No control series was tested owing to the many variables involved and the many results reported from other centres using standard techniques of anaesthesia.

Accordingly, from 1968, all patients admitted for cataract extraction were anaesthetized according to the following principles:

(x) Adequate intramuscular premedication with pethidine and phenothiazine, the newer more potent synthetic analgesics, such as phenoperidine or fentanyl, being avoided.

(2) Intravenous injection by an assistant of a butyrophenone tranquillizer, droperidol, immediately before surgery, in a dose graded according to age, weight, and response to the narcotics already administered.

(3) Adequate local analgesia and akinesia, using established techniques.

(4) Maintenance of adequate ventilation and airway during the operation, particular attention being paid to keeping towels away from the nose and mouth and making the patient as comfortable as possible during the operation.

(5) Monitoring and recording of blood pressure, pulse rate and rhythm, and level of consciousness at each of the above stages and for 4 hours postoperatively.

\section{Method}

One hour preoperatively the patient is given an intramuscular injection of pethidine, in a dose varying from 50 to $150 \mathrm{mg}$., depending on size, sex, age, and general physical condition, together with phenergan $25 \mathrm{mg}$.

In the theatre, intravenous droperidol is given slowly over a period of one minute, the dose varying from 3 to $5 \mathrm{mg}$. depending on the degree of sedation already achieved by the premedication.

The local anaesthetic, $10 \mathrm{ml}$. I per cent. lignocaine with adrenaline $1: 80,000$ in which 1,000 units of hyalase are dissolved, is then gently infiltrated in the usual manner to block the facial nerve and the ciliary ganglion. By this time the patient is generally relaxed and drowsy. During the time it takes the surgeon to "scrub up" after the instillation of the local anaesthetic, this drowsiness deepens to sleep and the respiration rate and pulse rate settle to steady levels. The blood pressure drops slightly but the patient remains cooperative and responsive to simple directions or instructions.

When the operation, which normally takes about 30 minutes, is over, it is easy to rouse the patient by speaking to him and, at this stage, most express their thanks.

\section{Material}

The sex and age distributions of the roo patients tested ( 34 male and 66 female) is fairly typical of any similar series of patients coming for lens extraction (Fig. I). Most of them, being elderly, had at least one chronic, complicating illness which, if not a contraindication for general anaesthesia, made 
them a poorer risk and might influence the decision whether to extract the lens or not. Only 21 of the roo patients could be described as "fit" (Fig. 2).

Nearly all the male patients received a dose of $5 \mathrm{mg}$. droperidol, while the female patients and the very old received a smaller dose (Fig. 3 ).
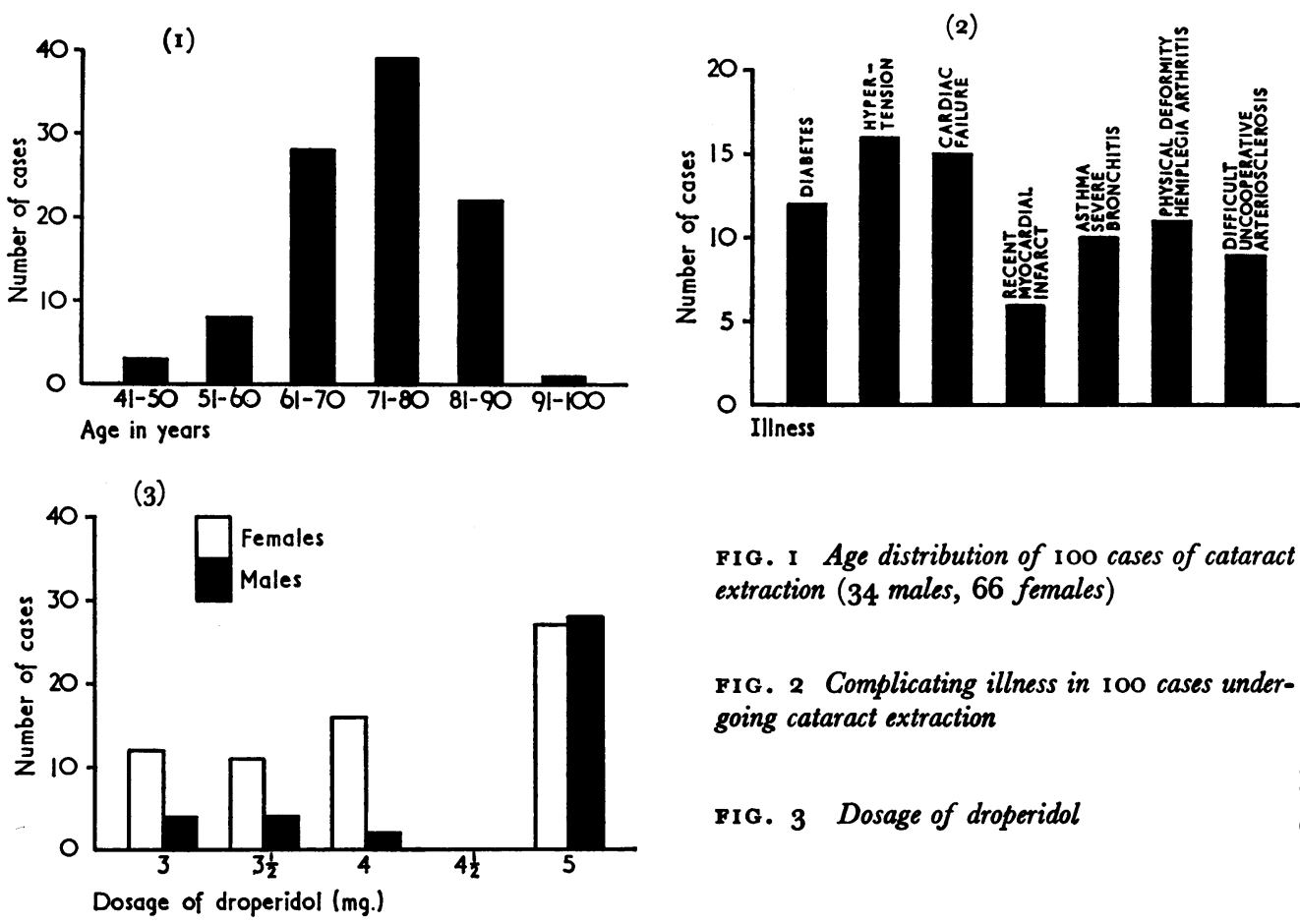

FIG. I Age distribution of 100 cases of cataract extraction (34 males, 66 females)

FIG. 2 Complicating illness in 100 cases undergoing cataract extraction

FIG. 3 Dosage of droperidol

All extractions were performed by the same surgeon, using a straightforward technique with preplaced corneo-scleral sutures and section by knife and scissors, the lens being simply expressed or tumbled, after the use of a zonulysin. The intracapsular method was used in 97 cases, and the extracapsular in two because of high myopia. One intumescent lens ruptured on delivery.

\section{Results}

The drop in the radial pulse rate 15 minutes and 1 hour after the intravenous injection of droperidol is shown in Fig. 4. Two patients developed bradycardia after the injection of droperidol; one responded immediately to intravenous atropine $0.3 \mathrm{mg}$. and gave no further cause for alarm. The other remained depressed and hypothermic for 12 hours,

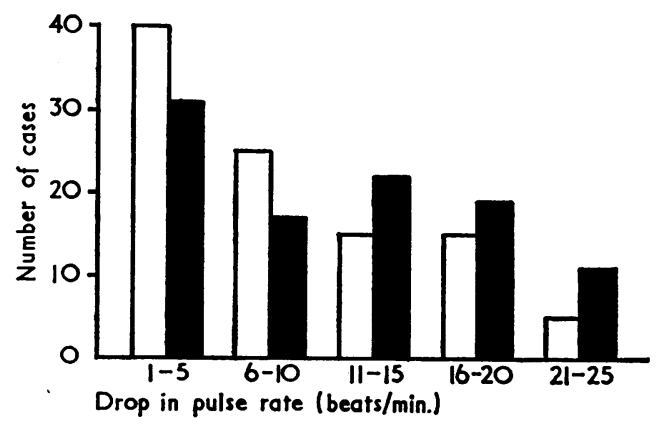

FIG. 4 Radial pulse rate per minute before operation and 15 minutes $\square$ and I hour $\square$ after injection 
and required intravenous fluids and stimulants before she recovered. Investigation showed this patient to be suffering from myxoedema which had not been suspected until this reaction occurred.

The fall in systolic blood pressure 15 minutes and one hour after the intravenous injection of droperidol is shown in Fig. 5. Most of the large falls occurred in patients in whom the pressure was raised preoperatively, and this gave no cause for alarm.

The fall in diastolic blood pressure is similarly shown in Fig. 6.

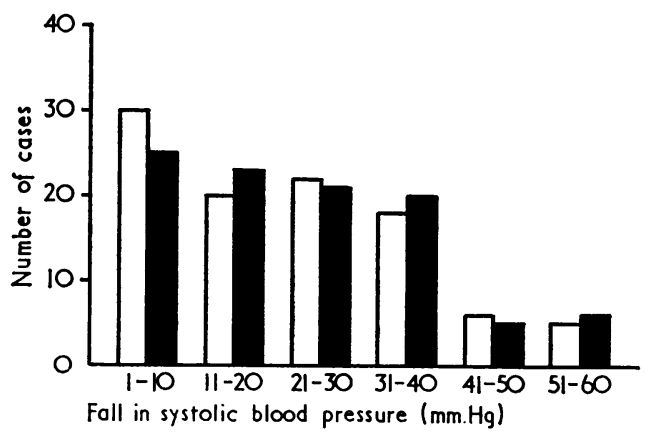

FIG. 5 Systolic blood pressure before operation and 15 minutes $\square$ and I hour $\square$ after injection

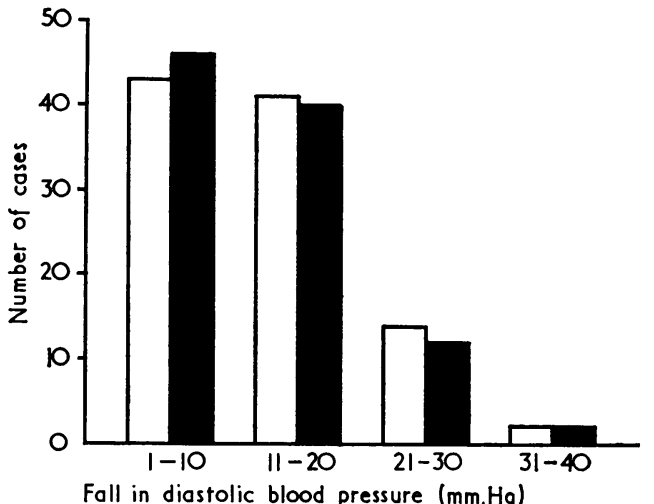

FIG. 6 Diastolic blood pressure before operation and I 5 minutes $\square$ and I hour $\square$ after injection

Fig. 7 illustrates three opinions on the level of consciousness of the patient during the operation. From the surgeon's point of view, 96 were adequately anaesthetized, cooperative, and relaxed, and haemorrhage at operation or postoperatively was less than under general anaesthesia with tracheal intubation. The remaining four were difficult to control because analgesia was initially poor, and once cooperation was lost, proved difficult to regain. This was probably because the preoperative dose of pethidine was too small. All four cases came at the beginning of the series. In only one case did vitreous loss occur because of the distress of the patient and, luckily, this did not affect the final visual result.

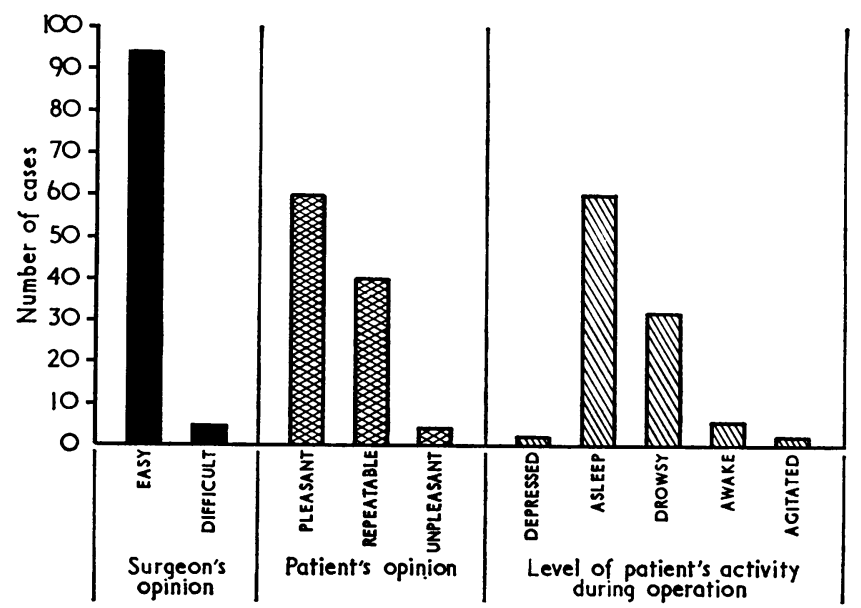

FIG. 7 Opinions of surgeon, patient, and anaesthetist on the effectiveness of droperidol 
From the patients' point of view, nearly all were enthusiastic supporters of this method, and, where necessary, were quite happy to have the second eye operated on in the same way. The one patient who was most vehement about not liking the procedure was recorded as being fast asleep during the whole of the operation which was notable for its lack of drama or complications.

Fig. 8 shows the visual acuity in the affected eye, before and after operation.

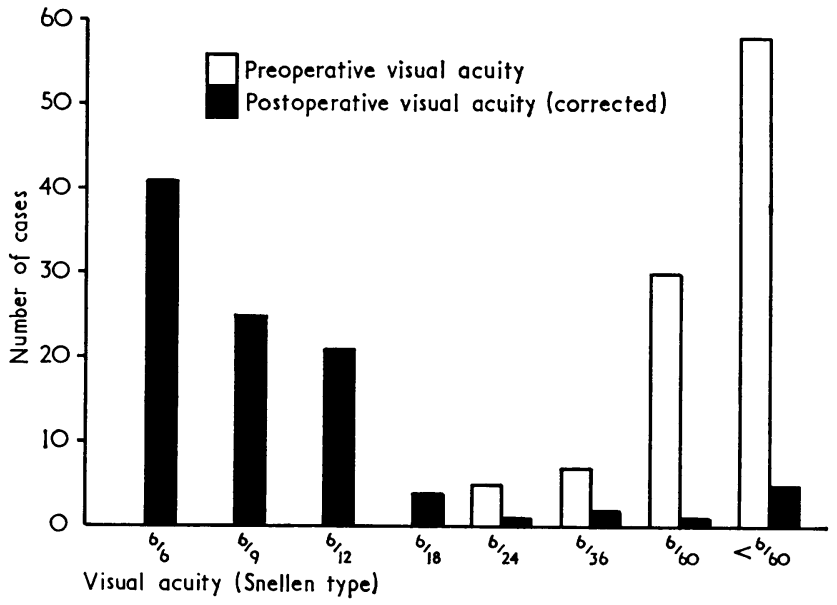

FIG. 8 Visual acuity in the affected eye before and after operation

Postoperatively there was not one case of nausea or vomiting, disorientation, or restlessness. Most of the patients passed into a normal sleep for a few hours on being returned to their beds, and only one had to have a postoperative sedative or analgesic. This effect is particularly remarkable because the series included four patients who were mentally subnormal, and eight others who could be classified as difficult and uncooperative because of senile arteriosclerosis or personality defects associated with old age.

\section{Summary}

A simple method of anaesthesia in cataract surgery is described, in which premedication with pethidine and phenergan is combined with a neuroleptic, droperidol, given intravenously, immediately before operation under local anaesthetic block. In Ioo cases admitted to a general hospital for cataract surgery only minor difficulties or hazards were found, and this method would appear to have considerable advantages for both the surgeon and the patient, particularly with the poor risk cases likely to be encountered in cataract surgery in the elderly.

I should like to thank Mr. P. A. Gardiner, consultant ophthalmologist, Barnet General Hospital, for his advice, encouragement, and permission to publish these cases, and Dr. M. F. Corfield, consultant anaesthetist, for his helpful criticisms.

\section{References}

BROWN, A. s. (1964) Anaesthesia, r9, 70

holderness, M. C., GHASe, P. E., and Dripps, R. D. (1963) Anesthesiology, 24336

LABORIT, H., and HUGUENARD, P. (1954) "Pratique de l'hibernothérapie en chirurgie et en médicine". Masson, Paris

moran, J. H., and marshall, B. m. (rg66) Canad. Anaesth. Soc. F., r3, 272

NILSSON, E., and JANSSEN, P. (I96I) Acta anaesth. scand., 5, 73

rollason, w. N., and sutherland, J. s. (1963) Anaesthesia, 18, 16

STALLARD, H. B. (I965) “Eye Surgery", 4th ed., p. 70. Wright, Bristol. 